\title{
A sensitive and compact optical detector based on lock-in amplification
}

\author{
Andrew J. Harvie*, Surendra K. Yadav, John C. de Mello* \\ Department of Chemistry, NTNU, Trondheim, Norway \\ email: andrew.j.harvie@ntnu.no and john.demello@ntnu.no
}

\begin{abstract}
:
We report a sensitive, fixed-wavelength, lock-in-based optical detector built from a light-emitting diode, two colour filters, a photodetector, a small number of discrete analogue components, and a low-cost microcontroller development board. We describe the construction, operating principle, use and performance of the optical detector, which may be used for both absorption- and fluorescencemeasurements in either a $10-\mathrm{mm}$ pathlength cuvette or a low-volume $(<100 \mu \mathrm{l})$ flow-cell. For illustrative purposes the detector is applied here to a cholesterol assay based on the enzyme-mediated conversion of (non-emissive) Amplex Red into the fluorescent dye resorufin, providing a detection limit of $\sim 200 \mathrm{nM}$ - some four orders of magnitude lower than the typical concentration of cholesterol in human serum. (The resorufin molecule itself is detectable down to concentrations of $\sim 20 \mathrm{nM}$ ). The system may be readily adapted to other biomolecules through a simple change of enzyme.
\end{abstract}

Keywords: Bioassays, fluorescence, 3D printing, open hardware, digital signal processing, lock-in

\section{Specifications table}

\begin{tabular}{|l|l|}
\hline Hardware name & Lock-In Luminometer \\
\hline Subject area & $\bullet \quad$ Chemistry and Biochemistry \\
\hline Hardware type & $\bullet \quad$ Measuring physical properties and in-lab sensors \\
\hline Closest commercial analogue & Luminometer \\
\hline Open Source Licence & MIT \\
\hline Cost of Hardware & $<\mathrm{f135}$ \\
\hline Source File Repository & $\underline{\text { https://doi.org/10.5281/zenodo.5106523 }}$ \\
\hline
\end{tabular}

\section{Hardware in context}

The detection and quantitation of weak, slowly varying fluorescence signals from trace concentrations of chromophores is an essential task in many fields of science. Important examples include: biochemistry, where low-concentration biomolecules are quantified using fluorogenic assays; [1] synthetic chemistry, where reaction kinetics are often determined by monitoring changes in fluorescence intensity due to consumed or generated fluorophores; [2] and environmental science, where fluorescence is used to detect and quantify pollutants, either directly or indirectly via their interaction with probe dyes. [3-5]

In common with other optical signals, fluorescence is commonly monitored using a photodiode coupled to a sensitive current meter, with the current generated by the photodiode typically being proportional to the intensity of the incident light. At low fluorophore concentrations, the weak fluorescence signal is 
frequently masked by noise and/or strong background signals, preventing reliable detection and measurement. In such circumstances, the fluorescence signal may often be extracted by modulating the light-source (and therefore the fluorescence signal) at a fixed frequency $f_{\text {mod, }}$ and using a signal processing technique known as "lock-in" detection [6] to eliminate unwanted interferences at other frequencies. The lock-in technique is simple to implement and, as shown here, can give good performance even with inexpensive hardware, making it an attractive choice for low-cost, high sensitivity fluorescence detection.

In this manuscript we describe a sensitive, fixed-wavelength, lock-in-based fluorescence detector built from a light-emitting diode (LED), two colour filters, a photodetector, a small number of discrete analogue components, and a low-cost microcontroller development board. We describe the construction, operating principle, use and performance of the fluorescence detector, which allows measurements to be carried out in a $10-\mathrm{mm}$ pathlength cuvette or a low volume $(<100 \mu \mathrm{l})$ flow-cell. For illustrative purposes the detector is applied here to a cholesterol assay based on the enzyme-mediated conversion of (nonemissive) Amplex Red into the fluorescent dye resorufin.[1] The system may be readily adapted to other biomolecules through a simple change of enzyme.

\section{Hardware description}

The hardware (see photographs in Fig. 1) comprises three main parts: (i) a printed circuit board (PCB), onto which all electronic components except the LED light-source are mounted; (ii) a 3D-printed housing for the LED, optical filters and sample; and (iii) a 3D-printed base-plate that holds the optics and PCB together. The PCB contains power management circuitry, an LED driver, an amplified photodiode to detect the emitted light, analogue circuitry for signal conditioning, and a microcontroller development board for carrying out analogue to digital conversion and lock-in detection.
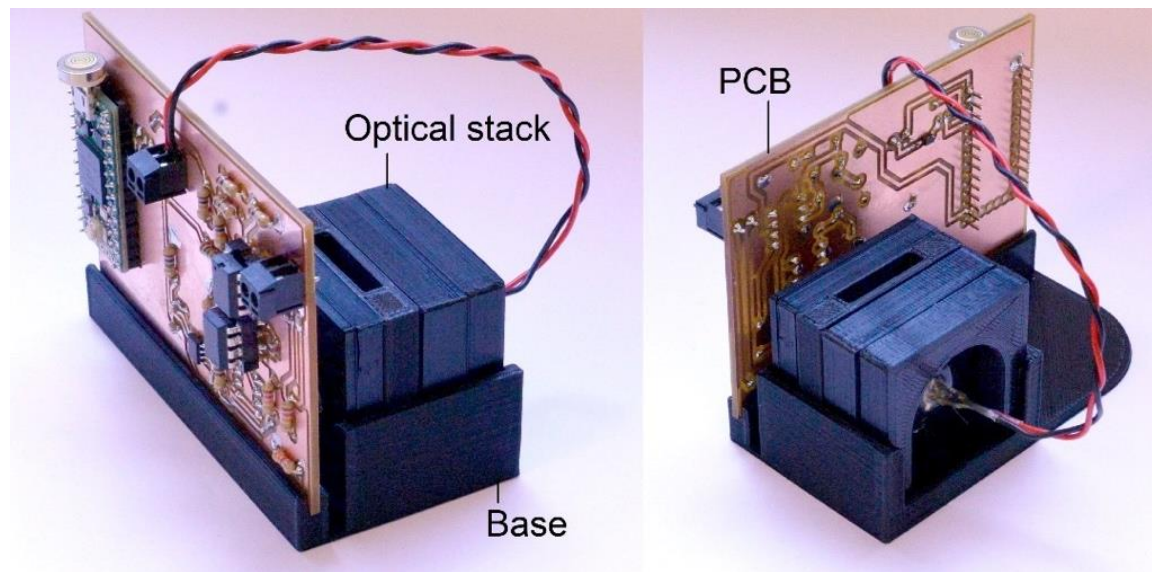

Fig. 1. Rear- and front-facing photographs of assembled fluorimeter. The device consists of three main parts: a PCB for the detection electronics, a 3D-printed housing for the optical components, and a 3D-printed base-plate for mechanical support. An LED inside the housing is used as a light-source, while an amplified photodiode mounted on the rear side of the PCB is used as a detector. 


\section{Optical detection}

The optical configuration is shown schematically in Fig. 2. Excitation light from the LED passes through a short-pass filter, which absorbs long-wavelength photons that overlap spectrally with the fluorophore emission. The filtered light from the LED passes through a detection zone containing the sample under test, where it is partially absorbed and reradiated by the fluorophores. The emergent light from the sample - a mixture of unabsorbed light from the LED and longer-wavelength light from the emitting fluorophores - passes through a long-pass filter, which heavily attenuates the excitation light but allows the emitted light to pass and strike the photodetector. The sample is introduced into the detection zone using a 10-mm cuvette or a low-volume $(<100 \mu \mathrm{l})$ flow cell. Interchangeable 3D-printed holders allow for easy swapping between cuvette- and flow-based detection. (The greater sample volume of the cuvette typically provides slightly better sensitivity but requires sample volumes of at least $2 \mathrm{ml}$ compared to $\sim 100 \mu \mathrm{l}$ for the flow cell). The holders may be readily adapted to other sample formats.

To reduce size, minimise the parts count, and simplify assembly, we do not use discrete focussing optics to guide the exciting or emitted light, although the chosen LED has a focussing lens integrated within its housing. If required, additional lenses could be straightforwardly incorporated into the housing for better light management and enhanced sensitivity. By replacing the short- and long-pass filters with band-pass filters matched to the emission peak of the LED, the system may be straightforwardly adapted to absorption-based measurements. (The ability to switch between fluorescence- and absorption-based measurements is the reason why we have chosen to arrange the light-source and detector in a face-toface configuration rather than at $90^{\circ}$ to one another, although it would be simple to adapt the system to orthogonal fluorescence detection if required).

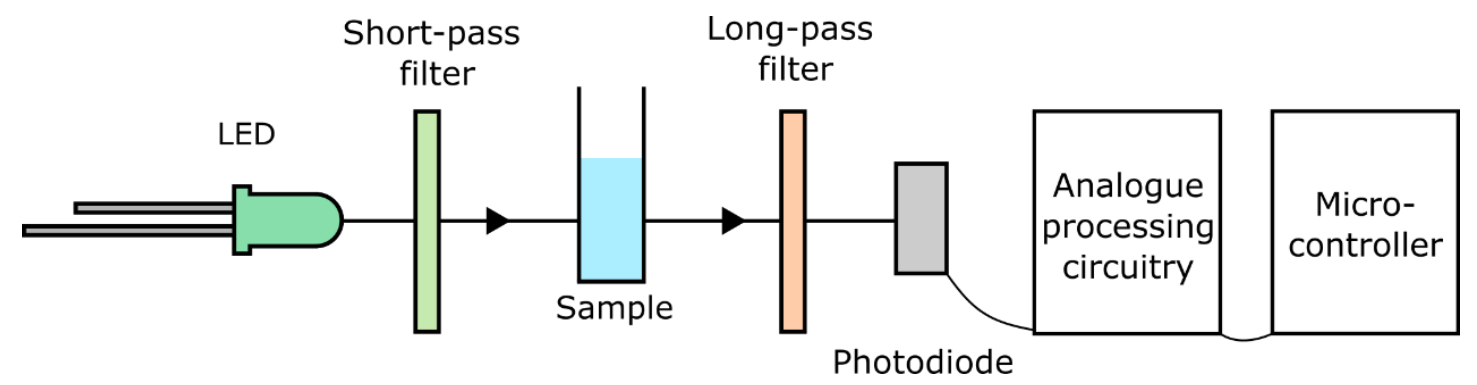

Fig. 2. Simplified schematic of optical setup. Light from an LED passes through a short-pass filter and strikes the sample, which is held in a $10-\mathrm{mm}$ pathlength cuvette or a simple flow cell. Emitted light from the sample plus transmitted light from the LED strikes a long-pass filter that allows only the emitted light to pass and reach the amplified photodiode. The signal from the photodiode is pre-processed using analogue circuitry and then passed to a microcontroller development board for analogue to digital conversion and digital signal processing.

For the purposes of cholesterol detection, the LED is chosen to have an emission peak at $525 \mathrm{~nm}$, which coincides with the absorption band of the resorufin dye used in the enzymatic assay. The Full Width Half Maximum (FWHM) of the LED's emission spectrum is approximately $25 \mathrm{~nm}$, but a long-wavelength tail extends beyond $570 \mathrm{~nm}$ (Fig. S1a). The emission tail is attenuated by the short-pass filter, which has a cutoff at $550 \mathrm{~nm}$ (Fig. S1b). The emission band of resorufin lies predominantly in the range $550-650 \mathrm{~nm}$, with a weak tail that extends to longer wavelengths (Fig. S1a). The long-pass filter has a cut-on at $570 \mathrm{~nm}$ 
(Fig. S1b) and therefore blocks most of the short-pass-filtered excitation light, while efficiently passing the emitted light. The LED is modulated at $\sim 410 \mathrm{~Hz}$, using a 30-mA constant-current driver circuit (see Fig. 3) that is toggled on and off with a $50 \%$ duty cycle using a digital output pin of the microcontroller. For the 30-mA square-wave modulation used here, the time-averaged output power of the LED is just over $1 \mathrm{~mW}$. The amplified silicon photodiode (OPT101) has a broadband optical response from 400 to $1000 \mathrm{~nm}$, which fully covers the emission band of resorufin (Fig. S1C). The set-up may be readily adapted to assays based on other dye molecules by swapping the LED and optical filters as needed.

\section{Analogue signal processing}

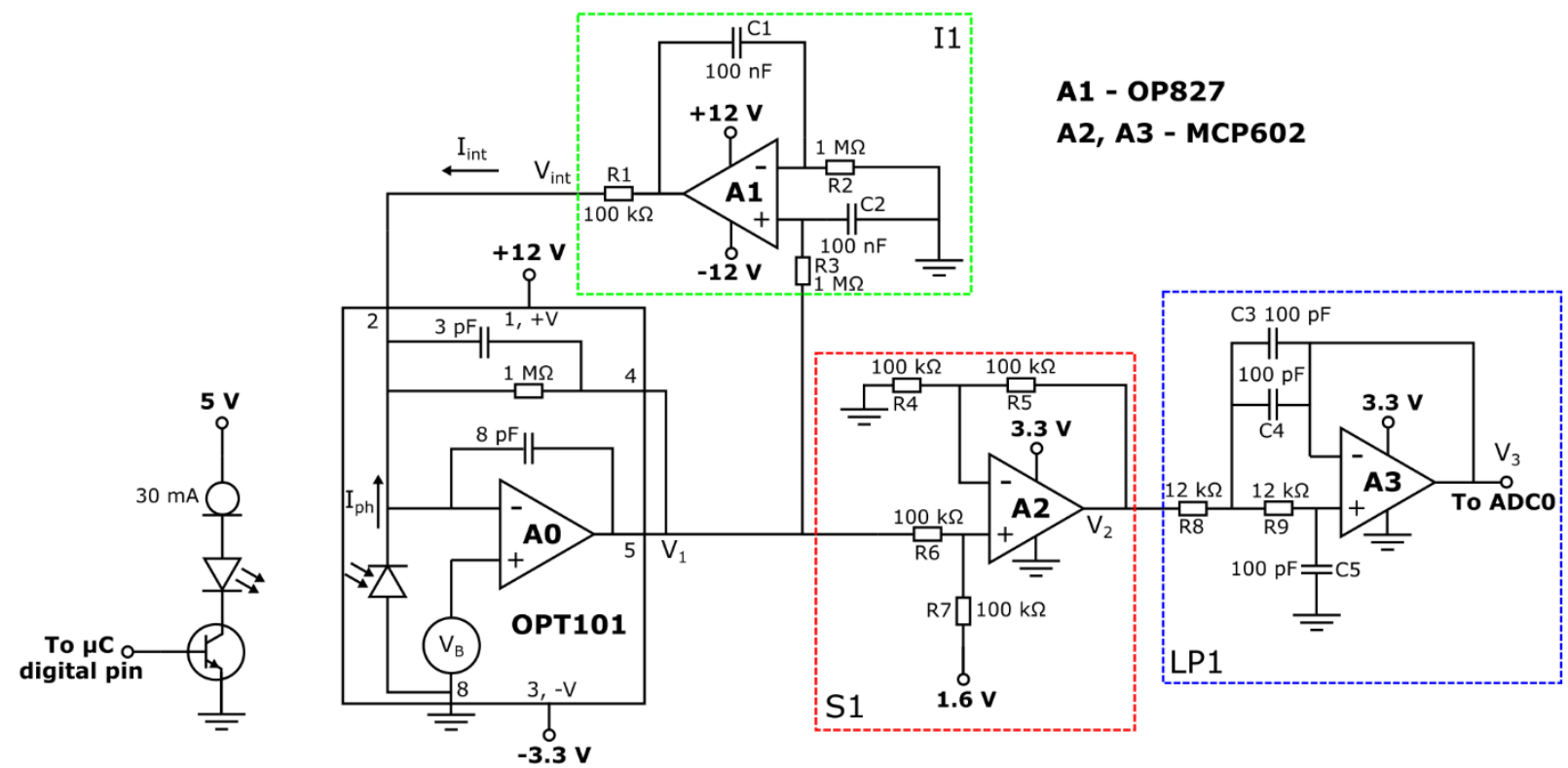

Fig. 3. Schematics of LED driver and analogue conditioning circuit. The LED is connected in series with a 30-mA constant current diode and an NPN transistor; the base terminal of the transistor is connected to a digital output pin of the microcontroller, which switches the current flow on and off at a frequency of $\sim 410 \mathrm{~Hz}$ and a duty cycle of $50 \%$. The optical signal is detected using an amplified photodiode (OPT101). The DC output $V_{1}$ from the amplified photodiode is passed through a non-inverting integrator (I1, green box) that generates a positive output current $I_{\text {int }}$ at the summing terminal of A0 (Pin 2 of the OPT101), cancelling the negative DC photocurrent due to ambient light and hence reducing the DC component of $V_{1}$ to zero. $V_{1}$ is passed to a non-integrating amplifier that adds a constant DC offset of $1.6 \mathrm{~V}$, yielding an output voltage $V_{2}$ that spans the range 0 to $3.2 \mathrm{~V}\left(\mathrm{~S} 1\right.$, red box). Finally, $V_{2}$ is passed to an active second-order low-pass filter (LP1, blue box) that suppresses noise and high-frequency components above $94 \mathrm{kHz}$.

The amplified photodiode (OPT101) is used in dual-supply mode with a transimpedance gain of $-1 \mathrm{~V} / \mu \mathrm{A}$. The output $V_{1}$ of the amplified photodiode feeds into a three-stage signal-conditioning circuit that prepares the signal for read-out by the microcontroller's built-in analogue-to-digital converter ADCO (which accepts input voltages in the range 0 to $3.3 \mathrm{~V}$ ). The first stage of the conditioning circuit is a feedback-based compensation stage that removes any DC offset in $V_{1}$ due to ambient light or non-ideality of A0, allowing the OPT101 to operate under conditions of high ambient illumination without saturating ADC0. (Note, the compensation circuit treats all frequencies below $16 \mathrm{~Hz}$ as $D C$ and hence the modulation frequency must be substantially higher than $16 \mathrm{~Hz}$ for a reliable measurement. For the present set-up, we 
use a fixed modulation frequency of $\sim 410 \mathrm{~Hz}$ ). The second stage of the conditioning circuit is a summing amplifier (S1) that converts an input signal in the range $-1.6 \mathrm{~V}$ to $+1.6 \mathrm{~V}$ into an output signal in the range $0 \mathrm{~V}$ to $3.2 \mathrm{~V}$, allowing it to be sampled by ADCO. The third stage is a unity-gain low-pass filter with a cutoff frequency of $94 \mathrm{kHz}$, slightly less than half the $200-\mathrm{kHz}$ sample rate used by ADCO. The low-pass filter attenuates noise and interferences above the 100-kHz Nyquist frequency of ADC0, preventing sampling artefacts due to aliasing. Further details of the conditioning circuit are provided in the Supporting Information.

\section{Digital signal processing}

Analogue to digital conversion and digital signal processing is carried out on a Teensy 4.0 microcontroller development board (PJRC). The Teensy 4.0 has a fast 600-MHz ARM processor with on-board Floating Point Unit (FPU), allowing it to efficiently carry out the calculations needed to implement digital lock-in detection. In brief, a sampled periodic signal $S$ of frequency $f_{0}$ is multiplied by sinusoidal and cosinusoidal reference signals of matching frequency $f_{0}$, and the resulting output signals are passed through separate digital low-pass filters to remove non-DC components (see the block diagram in Fig. 4). Following low-pass filtering we obtain two scalar outputs $X$ and $Y$ derived from the two reference signals. $X$ and $Y$ are vectorially combined to obtain an output signal $R=\sqrt{ }\left(X^{2}+Y^{2}\right)$ that is directly proportional to the amplitude of $S$. (Formally, $R$ corresponds to the amplitude of the first harmonic of $S$ ). The process repeats every time a new sample is acquired, so the lock-in outputs $X, Y$ and $R$ are updated at the sample rate of the ADC. $R$ is affected only by input signals that are very close in frequency to $f_{0}$ so the target signal $S$ may be reliably measured even if it is corrupted by significant noise and/or interferences at other frequencies. A detailed description of the lock-in method is provided in the Supporting Information.

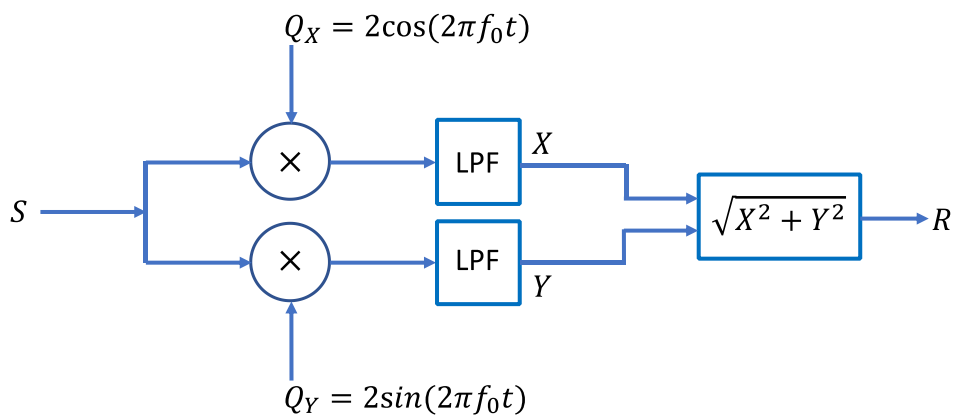

Fig. 4. Block diagram illustrating lock-in procedure. An input signal $S$ of frequency $f_{0}$ is passed into two separate channels. In the first channel $S$ is multiplied by a reference signal of the form $2 \cos \left(2 \pi f_{0} t\right)$ and passed through a low-pass filter (LPF), yielding an intermediate output $X$, while in the second channel it is multiplied by a reference signal of the form $2 \sin \left(2 \pi f_{0} t\right)$ and passed through a second low-pass filter, yielding an intermediate output $Y$. $X$ and $Y$ are vectorially combined to yield a final output $R=$ $\sqrt{X^{2}+Y^{2}}$ that is proportional to the amplitude of input signal $S$.

Computationally, the lock-in procedure is carried out as follows. An incoming analogue signal of frequency $f_{0}$ (together with noise and interfering signals at other frequencies) is sampled at discrete times $t(1)$, $t(2), t(3) \ldots$ where the time interval $\Delta t$ between successive measurements is fixed at $5 \mu \mathrm{s}$. Timing errors are minimised by choosing $f_{0}$ to be an exact integer fraction of the sample rate $f_{\mathrm{s}}$, i.e. $f_{0}=f_{\mathrm{s}} / \mathrm{m}=$ $1 /(m \Delta t)$ where $m$ is an integer. Here, we choose a sample rate of $200 \mathrm{kHz}$ and an $m$-value of 488 , which 
corresponds to $f_{0}=409.84 \mathrm{~Hz}$. The sample values $S(1), S(2), S(3)$... are obtained from the ADC as 12 bit integers and stored as 64-bit doubles. All calculations are carried out at 64-bit precision using the microcontroller's onboard FPU. Immediately after each sample $S(n)$ has been acquired, the two reference signals $Q_{x}(n)$ and $Q_{y}(n)$ are updated as follows:

and

$$
Q_{x}(n)=\cos \left(2 \pi f_{0} n \Delta t\right)=\cos (2 \pi n / m)
$$

$$
Q_{y}(n)=\sin \left(2 \pi f_{0} n \Delta t\right)=\sin (2 \pi n / m)
$$

where the sample number $n=1,2,3 \ldots$ The LED driver is toggled on or off every $m / 2$ steps, i.e. whenever $Q_{y}(n)=0$, resulting in a fluorescence signal that is phase-locked to the reference signals and of identical frequency. (Note, the exact phase of the fluorescence signal relative to the reference signals depends on the turn-on time of the LED driver and varies according to the modulation frequency. The use of two reference signals that differ in phase by $90^{\circ}$ corrects for the effects of the slow LED turn-on).

Multiplying $Q_{x}(n)$ and $Q_{y}(n)$ by $S(n)$ we obtain two (unfiltered) intermediate outputs $X_{0}(n)$ and $Y_{0}(n)$ :

and

$$
X_{0}(n)=Q_{x}(n) S(n)
$$

$$
Y_{0}(n)=Q_{y}(n) S(n)
$$

The low-pass filtering step is carried out by exponentially averaging $X_{0}(n)$ and $Y_{0}(n)$, [7] which yields two intermediate outputs $X_{1}(n)$ and $Y_{1}(n)$ :

and

$$
X_{1}(n)=X_{1}(n-1)+\alpha\left[X_{0}(n)-X_{1}(n-1)\right]
$$

$$
Y_{1}(n)=Y_{1}(n-1)+\alpha\left[Y_{0}(n)-Y_{1}(n-1)\right]
$$

where $X_{1}(n-1)$ and $Y_{1}(n-1)$ are the previous values of $X_{1}$ and $Y_{1}$ and $\alpha$ is a weighting coefficient that determines the cut-off frequency of the exponential filter. ( $X_{1}$ and $Y_{1}$ are arbitrarily set to zero prior to the first sample). $\alpha$ is set to a constant value of $7.85 \times 10^{-6}$, which corresponds to a cut-off frequency $f_{c}$ of $0.25 \mathrm{~Hz}$ and a time-constant $\tau$ of $0.63 \mathrm{~s}$.[7] The exponential filtering stage is repeated using the same $\alpha$ value to provide better attenuation of non-DC interferences and hence a 'cleaner' solution:

and

$$
X_{2}(n)=X_{2}(n-1)+\alpha\left[X_{1}(n)-X_{2}(n-1)\right]
$$

$$
Y_{2}(n)=Y_{2}(n-1)+\alpha\left[Y_{1}(n)-Y_{2}(n-1)\right]
$$

Finally, $X_{2}(n)$ and $Y_{2}(n)$ are combined vectorially to obtain a single scalar output $R_{2}(n)$ :

$$
R_{2}(n)=\sqrt{X_{2}(n) X_{2}(n)+Y_{2}(n) Y_{2}(n)}
$$


$R_{2}(n)$ is proportional to the amplitude of the first harmonic of the target signal $S$ (see Supporting Information) which, in practical terms, means it is proportional to the average fluorescence intensity of the sample under test (plus a background contribution from residual LED light that leaks through the longpass filter). Interferences from spurious signals at other frequencies are greatly attenuated by the lock-in algorithm (see Supporting Information).

The microcontroller sends out a new $R_{2}$ value over its universal serial bus (USB) interface every $0.1 \mathrm{~s}$ for remote analysis on a PC or other hardware. For a reliable measurement, it is advisable to wait 10 time constants after switching on the sensor, loading a new sample, or changing a parameter for the lock-in output to stabilise. Hence, for the $0.63 \mathrm{~s}$ time constant used here, a wait time of 6-7 s is recommended. A simple PC-based software front-end is provided for reading, plotting and saving data (see operating instructions).

\section{Power management}

The PCB has two sets of bipolar power rails at $\pm 3.3 \mathrm{~V}$ and $\pm 12 \mathrm{~V}$. The $\pm 3.3 \mathrm{~V}$ rails are powered by the Teensy's built-in 3.3 V supply (which in turn is powered via the USB connection), using a TC7660 inverting charge pump IC on the PCB to feed the $-3.3 \mathrm{~V}$ rail. The $\pm 12 \mathrm{~V}$ rails are derived from an external $24 \mathrm{~V}$ unipolar supply, using a resistive divider with its midpoint connected to the ground of the $3.3 \mathrm{~V}$ unipolar supply. The Teensy microcontroller and the (single-supply) MCP602 dual op-amp IC used in S1 and LP1 are operated at $+3.3 \mathrm{~V}$. The OP827 op-amp in the DC compensation circuit is operated at $\pm 12 \mathrm{~V}$, and the OPT101 amplified photodiode is powered using a combination of the $+12 \mathrm{~V}$ and $-3.3 \mathrm{~V}$ rails to allow for bipolar operation around $0 \mathrm{~V}$.

\section{Main features of optical detector at-a-glance:}

- Easy measurement of fluorescence in a flow-cell or 10-mm-pathlength cuvette

- Excellent rejection of ambient light, noise and other interferences

- High sensitivity and linearity over a wide range of intensities

- Applicable to a wide range of fluorogenic biochemical assays

- Modular design can be customised to accept a wide range of samples, LEDs and filters 


\section{Design files}

\begin{tabular}{|l|l|l|l|}
\hline Design file name & File type & Open source licence & Location of the file \\
\hline Base.stl & CAD file - 3D print & MIT Licence & $\underline{\text { https://doi.org/10.5281/zenodo.5106523 }}$ \\
\hline EmissionPlate.stl & CAD file - 3D print & MIT Licence & $\underline{\text { https://doi.org/10.5281/zenodo.5106523 }}$ \\
\hline ExcitationPlate.stl & CAD file - 3D print & MIT Licence & $\underline{\text { https://doi.org/10.5281/zenodo.5106523 }}$ \\
\hline FlowCellHalf1.stl & CAD file - 3D print & MIT Licence & $\underline{\text { https://doi.org/10.5281/zenodo.5106523 }}$ \\
\hline FlowCellHalf2.stl & CAD file - 3D print & MIT Licence & $\underline{\text { https://doi.org/10.5281/zenodo.5106523 }}$ \\
\hline FlowCellHolder.stl & CAD file - 3D print & MIT Licence & $\underline{\text { https://doi.org/10.5281/zenodo.5106523 }}$ \\
\hline PDPlate.stl & CAD file - 3D print & MIT Licence & $\underline{\text { https://doi.org/10.5281/zenodo.5106523 }}$ \\
\hline CuvetteHolder.stl & CAD file - 3D print & MIT Licence & $\underline{\text { https://doi.org/10.5281/zenodo.5106523 }}$ \\
\hline RetainingClip.stl & CAD file - 3D print & MIT Licence & $\underline{\text { https://doi.org/10.5281/zenodo.5106523 }}$ \\
\hline CuvetteCap.stl & CAD file - 3D print & MIT Licence & $\underline{\text { https://doi.org/10.5281/zenodo.5106523 }}$ \\
\hline PCB.brd & CAD file - PCB & MIT Licence & $\underline{\text { https://doi.org/10.5281/zenodo.5106523 }}$ \\
\hline PCBGerbers.zip & CAM files - PCB & MIT Licence & $\underline{\text { https://doi.org/10.5281/zenodo.5106523 }}$ \\
\hline FluorescenceSensor.ino & Microcontroller firmware & MIT Licence & $\underline{\text { https://doi.org/10.5281/zenodo.5106523 }}$ \\
\hline Sensorlnterface.py & Software frontend & MIT Licence & $\underline{\text { https://doi.org/10.5281/zenodo.5106523 }}$ \\
\hline widget.ui & Software frontend component & MIT Licence & $\underline{\text { https://doi.org/10.5281/zenodo.5106523 }}$ \\
\hline communicator.py & Software frontend component & MIT Licence & $\underline{\text { https://doi.org/10.5281/zenodo.5106523 }}$ \\
\hline
\end{tabular}

Table 1 - Summary of design files

\begin{tabular}{|c|c|}
\hline Base.stl & STL model file for the 3D-printed base. \\
\hline $\begin{array}{l}\text { EmissionPlate.stl } \\
\text { ExcitationPlate.stl } \\
\text { FlowCellHolder.stl } \\
\text { PDPlate.stl } \\
\text { RetainingClip.stl }\end{array}$ & $\begin{array}{l}\text { STL model files for the 3D-printed components in the optical } \\
\text { stack (see Build Instructions for further details). }\end{array}$ \\
\hline $\begin{array}{l}\text { FlowCellHalf1.stl } \\
\text { FlowCellHalf2.stl }\end{array}$ & $\begin{array}{l}\text { STL model files for the 3D-printed parts used to construct } \\
\text { the liquid flow cell. }\end{array}$ \\
\hline CuvetteHolder.stl & STL model file for the 3D-printed cuvette holder. \\
\hline CuvetteCap.stl & $\begin{array}{l}\text { STL model file for an optional cap to protect the cuvette } \\
\text { from external light. }\end{array}$ \\
\hline PCB.brd & $\begin{array}{l}\text { BRD (Autodesk Eagle) format design files for the printed } \\
\text { circuit board. }\end{array}$ \\
\hline PCBGerbers.zip & $\begin{array}{l}\text { Collated Gerber and drill files for PCB manufacture. An } \\
\text { external manufacturer will likely require these files, } \\
\text { packaged within the .zip archive. }\end{array}$ \\
\hline FluorescenceSensor.ino & $\begin{array}{l}\text { Arduino source code for the microcontroller development } \\
\text { board. Arduino and Teensyduino must be installed on the } \\
\text { control PC. }\end{array}$ \\
\hline SensorInterface.py & $\begin{array}{l}\text { Python script for the software frontend. Requires installation } \\
\text { of Python 3.x or higher, the PyQt5, pyqtgraph and PySerial } \\
\text { libraries, plus widget.ui and communicator.py to run. }\end{array}$ \\
\hline
\end{tabular}

Table 2 - Design file description 


\section{Bill of Materials}

\begin{tabular}{|c|c|c|c|c|}
\hline Item & $\begin{array}{l}\text { Unit } \\
\text { cost }\end{array}$ & $\begin{array}{l}\text { Total } \\
\text { cost }\end{array}$ & Source & Material type \\
\hline Teensy $4.0 \times 1$ & $£ 18.50$ & $£ 18.50$ & $\begin{array}{l}\text { https://coolcomponents.co.uk/products/te } \\
\text { ensy-4-0-usb-development-board }\end{array}$ & Other \\
\hline PCB $\times 1$ & $\sim_{f 2}$ & $\sim f 2$ & Self-made & Composite \\
\hline $\begin{array}{l}\text { 3D-printed parts: } \\
\text { Base } \times 1 \\
\text { Retaining clip } \times 1 \\
\text { Excitation plate } \times 1 \\
\text { Emission plate } \times 1 \\
\text { Photodiode plate } \times 1 \\
\text { Flow cell mount } \times 1 \\
\text { Flow cell halves } \times 2\end{array}$ & & $\sim_{f 2}$ & Self-made & Polymer \\
\hline 8-pin DIP socket $\times 1$ & $f 0.62$ & $f 0.62$ & $\begin{array}{l}\text { https://uk.rs-online.com/web/p/dil- } \\
\text { sockets/0813115/ }\end{array}$ & Other \\
\hline TC7660EPA charge pump IC $\times 1$ & $£ 0.80$ & $£ 0.80$ & $\begin{array}{l}\text { https://uk.rs-online.com/web/p/voltage- } \\
\text { regulators/7747876/ }\end{array}$ & $\begin{array}{l}\text { Semiconductor } \\
\text { components }\end{array}$ \\
\hline MCP602-I/P op-amp IC × 1 & $£ 0.48$ & $£ 0.48$ & $\begin{array}{l}\text { https://uk.rs-online.com/web/p/op- } \\
\text { amps/3792572/ }\end{array}$ & $\begin{array}{l}\text { Semiconductor } \\
\text { components }\end{array}$ \\
\hline OPA827AID op-amp IC $\times 1$ & $f 12$ & $f 12$ & $\begin{array}{l}\text { https://uk.rs-online.com/web/p/op- } \\
\text { amps/7093267/ }\end{array}$ & $\begin{array}{l}\text { Semiconductor } \\
\text { components }\end{array}$ \\
\hline OPT101 amplified photodiode $\times 1$ & $f 6$ & f6 & $\begin{array}{l}\text { https://uk.rs- } \\
\text { online.com/web/p/photodetector- } \\
\text { amplifiers/1977465 }\end{array}$ & $\begin{array}{l}\text { Semiconductor } \\
\text { components }\end{array}$ \\
\hline $080510 \mu \mathrm{F}$ capacitor $\times 2$ & $£ 0.14$ & $f 0.28$ & $\begin{array}{l}\text { https://uk.rs-online.com/web/p/mlccs- } \\
\text { multilayer-ceramic-capacitors/9155225/ }\end{array}$ & Ceramic \\
\hline $0805100 \mathrm{nF}$ capacitor $\times 2$ & $£ 0.05$ & $£ 0.10$ & $\begin{array}{l}\text { https://uk.rs-online.com/web/p/mlccs- } \\
\text { multilayer-ceramic-capacitors/4646688/ }\end{array}$ & Ceramic \\
\hline 0805100 pF capacitor $\times 3$ & $£ 0.10$ & $£ 0.30$ & $\begin{array}{l}\text { https://uk.rs-online.com/web/p/mlccs- } \\
\text { multilayer-ceramic-capacitors/0391000/ }\end{array}$ & Ceramic \\
\hline $\begin{array}{l}\text { Resistors (through hole): } \\
3.3 \mathrm{k} \Omega \times 2 \\
12 \mathrm{k} \Omega \times 2 \\
10 \mathrm{k} \Omega \times 2 \\
100 \mathrm{k} \Omega \times 5 \\
1 \mathrm{M} \Omega \times 2 \\
\end{array}$ & & f1 & Generic & Any type \\
\hline $\begin{array}{l}\text { NSI45030AT1G } 30 \text { mA LED driver } \\
\times 1\end{array}$ & $£ 0.15$ & $£ 0.15$ & $\begin{array}{l}\text { https://uk.rs-online.com/web/p/display- } \\
\text { drivers/7377990P/ }\end{array}$ & $\begin{array}{l}\text { Semiconductor } \\
\text { components }\end{array}$ \\
\hline BC547B NPN transistor $\times 1$ & f0.14 & f0.14 & $\begin{array}{l}\text { https://uk.rs-online.com/web/p/bipolar- } \\
\text { transistors/7619828P/ }\end{array}$ & $\begin{array}{l}\text { Semiconductor } \\
\text { components }\end{array}$ \\
\hline $\begin{array}{l}\text { FGL570 } 570 \mathrm{~nm} \text { coloured long- } \\
\text { pass glass filter } \times 1\end{array}$ & £20.69 & $£ 20.69$ & $\begin{array}{l}\text { https://www.thorlabs.com/thorproduct.cf } \\
\text { m?partnumber=FGL570 }\end{array}$ & Glass optic \\
\hline $\begin{array}{l}\text { FES0550 } 550 \mathrm{~nm} \text { short-pass filter } \\
\times 1\end{array}$ & f60.47 & $£ 60.47$ & $\begin{array}{l}\text { https://www.thorlabs.com/thorproduct.cf } \\
\text { m?partnumber=FES0550 }\end{array}$ & Glass optic \\
\hline LED525L green LED $\times 1$ & f9.41 & f9.41 & $\begin{array}{l}\text { https://www.thorlabs.com/thorproduct.cf } \\
\text { m?partnumber=LED525L }\end{array}$ & $\begin{array}{l}\text { Semiconductor } \\
\text { components }\end{array}$ \\
\hline \multirow[t]{2}{*}{$3.5 \mathrm{~mm}$ PCB terminal block $\times 2$} & $£ 0.36$ & f0.72 & $\begin{array}{l}\text { https://uk.rs-online.com/web/p/pcb- } \\
\text { terminal-blocks/8971332/?sra=pstk }\end{array}$ & Other \\
\hline & & $£ 135.66$ & & \\
\hline
\end{tabular}

Table 3 - Bill of materials. Shaded rows indicate five costliest components. 
Comment on costs - We note that the system was assembled from parts that we already had to hand in our laboratory, and there is substantial scope for reducing the overall bill of materials through judicious component replacements. The shaded rows in Table 3 denote the five costliest items that together account for $88 \%$ of the bill of materials. At the time of writing, all items except the Teensy 4.0 microcontroller could be replaced by substantially cheaper alternatives without requiring any significant design changes. The 112 OPA827 op-amp used in the DC compensation circuit could be replaced by a variety of lower cost DC servo op-amps such as the Texas Instruments TL072CP, which costs approximately f1. The LED525L green LED could be replaced by a wide range of high brightness generic LEDs with similar optical output power and spectral characteristics, which are widely available for $£ 1$ or less. The long- and short-pass filters could be replaced by suitably chosen plastic colour filters at a cost of a few pounds. (Coloured 'gels' for stage lighting are a good source of low-cost colour filters, while home-made dyesensitised titania filters are an attractive option for high-sensitivity applications where filter fluorescence must be minimised[8,9]). By making these simple component substitutions, we consider it feasible to reduce the total bill of materials to around $£ 45$ without reducing the overall performance of the system. (Indeed switching to a more optimal pairing of short- and long-pass filters can be expected to improve performance, see Fig. S1b).

\section{Build instructions}

The PCB is designed with wide traces to allow easy fabrication from double-sided copper PCB blanks using a milling machine; alternatively PCB fabrication may be outsourced to a PCB service provider using the provided Gerber files. The locations, orientations and values of the components are shown in Fig. 5. A complete circuit diagram is provided in Fig. S2. All components other than the OPT101 amplified photodiode are soldered directly to the board; the OPT101 is mounted in an 8-pin DIP socket to simplify final assembly. The MCP602 and TC7660 may also be mounted in sockets, but this is not necessary. $\sim 10 \mathrm{~cm}$ leads connect the LED to the terminal block on the PCB.

All 3D-printed parts should be printed using black filament (polylactic acid [PLA] is suitable), using a layer thickness of $0.2 \mathrm{~mm}$ or less, and an infill of at least $10 \%$. Assembly of the instrument is straightforward, and is illustrated in Fig. 6a for the flow cell. The LED and OPT101 are glued into pre-formed recesses within their respective mounting plates using a small amount of hot glue. Next, the OPT101 is plugged into its socket on the PCB. The instrument is assembled by stacking the PCB and individual layers of the optical stack as shown in Fig. 6a, and slotting them - as a single unit - into the base; the optical stack is held together and retained in the base-plate by a friction fit. The flow-cell is formed using a $\sim 10-\mathrm{cm}$ length of 2-mm-OD, 1-mm-ID semi-transparent polytetrafluoroethylene (PTFE) tubing (228-4118, VWR) secured within the two 3D-printed halves using either hot glue or epoxy resin (see Fig. 6b). If required, the PTFE tubing could be replaced with more transparent fluorinated ethylene-propylene (FEP) tubing of the same dimensions, but this comes at a small cost premium. Fluidic connections to the internal tubing are made using nut-and-ferrule style Luer adaptors. The construction steps are almost identical for the cuvette holder, except the retaining clip is omitted to compensate for the greater depth of the cuvette holder. Side-by-side images of the two configurations are shown in Fig. 7. 


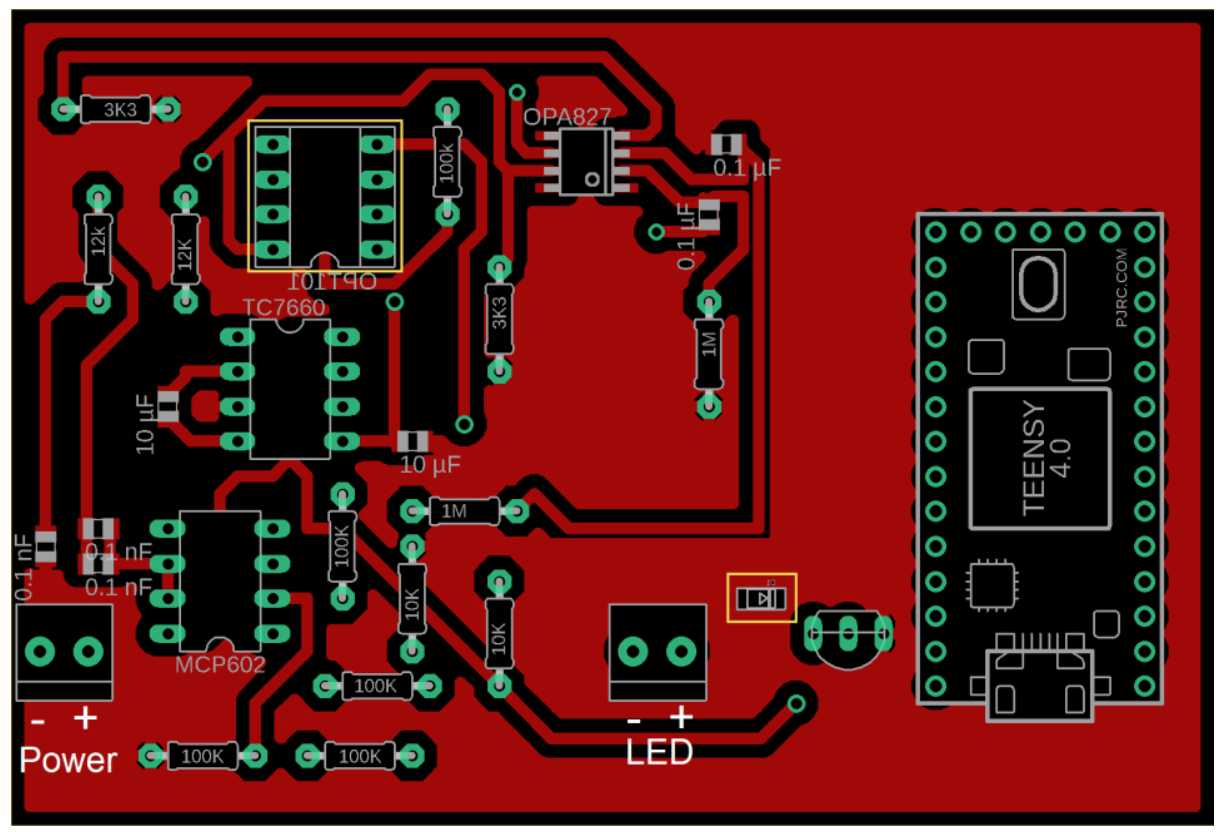

Fig. 5. Diagram of the top side of the $P C B$, showing component orientations and values. The two components soldered to the underside of the PCB (DIP socket for OPT101 and 30 mA LED driver) are marked with yellow boxes.
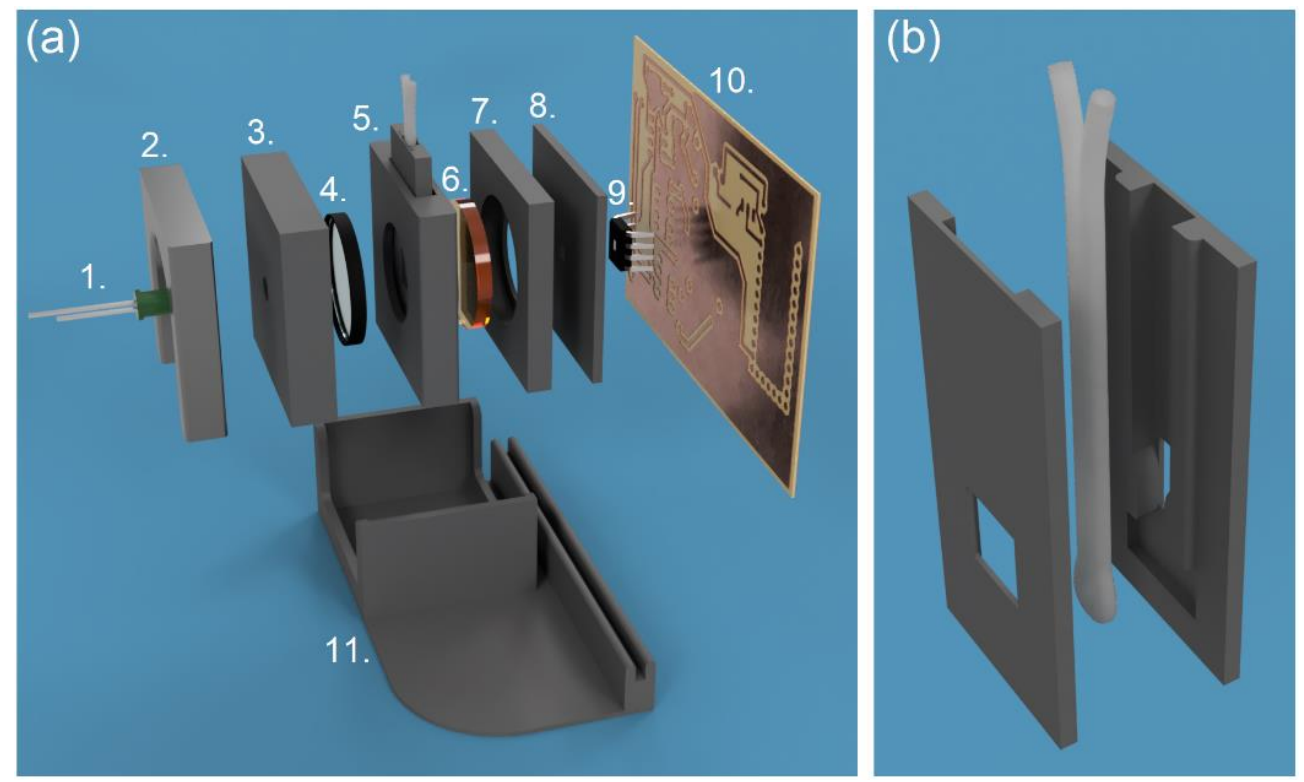

Fig. 6 (a) Exploded-view of detector assembly, showing: LED (1), retaining clip (2), mounting plate for LED and short-pass filter (3), short-pass filter (4), flow-cell assembly (5), long-pass filter (6), mounting plate for long-pass filter (7), mounting plate for amplified photodiode (8), amplified photodiode (9) and PCB (10). (The PCB is shown unpopulated for clarity). The unit is assembled by stacking together parts 1 to 10 and slotting into the base-plate (11). The ring-mounted short-pass filter (4) presses into its mounting plate (3) with a friction fit, whereas the long-pass filter is held in place via two protrusions that extend from the flow-cell assembly. The LED and photodiode package are glued into their respective mounting plates. (b) Exploded view of flow cell. The flow-cell is constructed from a short $(\sim 10 \mathrm{~cm})$, doubled-over length of 2-mm-OD, 1-mm-ID PTFE tubing glued between two 3D-printed retaining halves. 


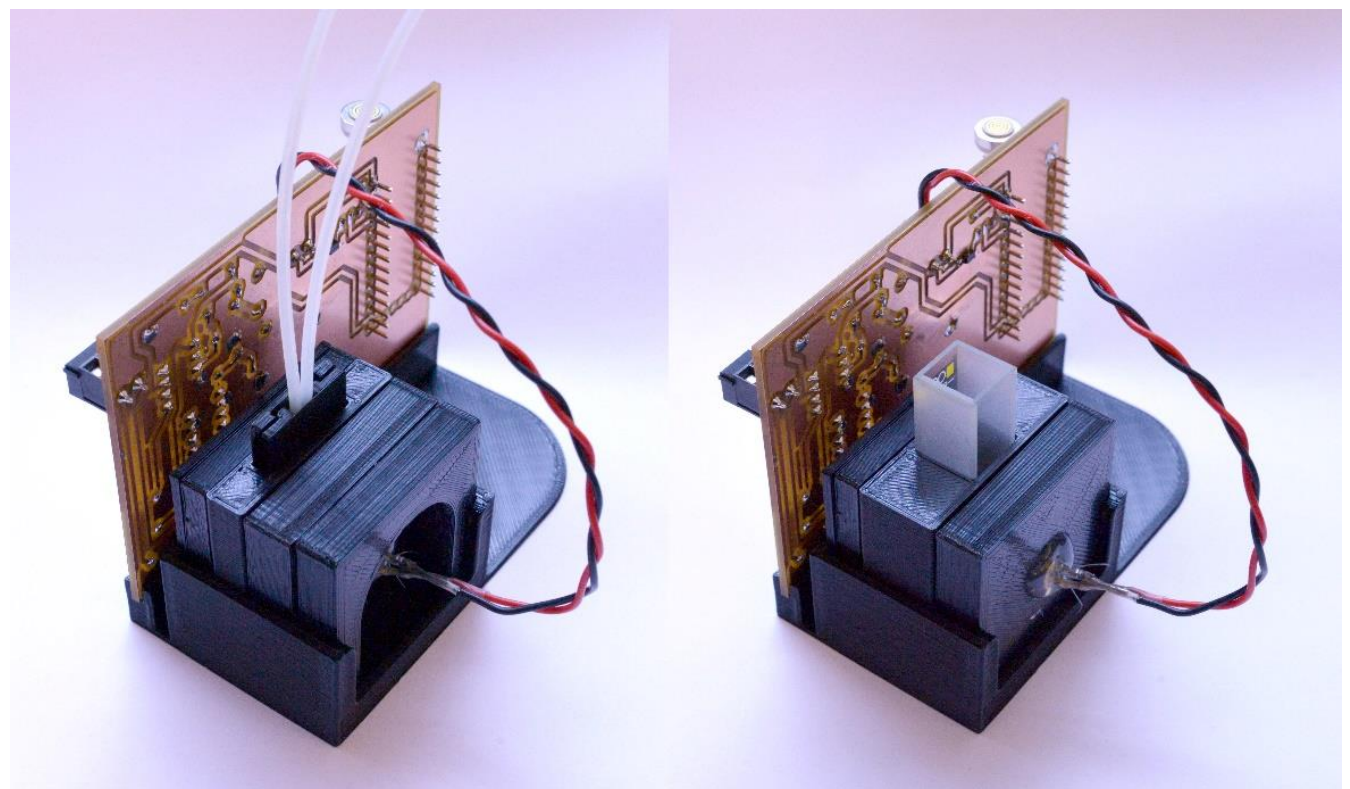

Fig. 7. Detector assembly configured for use with a flow cell (left) and a cuvette (right), with the corresponding sample holders installed. The retaining clip (part 2 in Fig. 6a) is omitted in the cuvette configuration to compensate for the extra thickness of the cuvette holder.

\section{Operating Instructions}

First, connect the PCB to a $20-24 \mathrm{~V}$ unipolar power supplying, using the terminal block labelled power in Fig. 5. Then select measurement settings within the firmware and flash the microcontroller. We recommend sticking to the default sample rate of $200 \mathrm{kHz}$ and the default modulation frequency of $\sim 410 \mathrm{~Hz}$, but if necessary these can be changed by modifying the values of Fsig and Fsample in the pre-processor definitions of the file "LockInFirmware.ino". The relevant locations are clearly commented.

Flashing of the firmware is most easily achieved using the Arduino IDE, which may be downloaded from https://www.arduino.cc/en/software. It is also necessary to download and install Teensyduino (https://www.pirc.com/teensy/td download.html) for compatibility with the Teensy 4.0. Flash the firmware by opening the firmware file "FluorescenceSensor.ino" within the Arduino IDE, connecting the device via a USB cable, and clicking the "Upload" button within the Arduino IDE. Once flashed, the microcontroller will output the measured lock-in amplitude $R_{2}$ at 0.1-s intervals, which can be read using the Arduino IDE's serial monitor, or using the software front-end described below. Typically, it takes several minutes for the output of an LED to stabilise after initial switch-on, and we recommend waiting at least 15 minutes before beginning measurements.

Measurements are most easily performed using the provided Python program. The software, "SensorInterface.py", was written in Python 3.8.8, and requires installation of PyQt5, pyqtgraph, and numpy. A screenshot of the interface is shown in Fig. 8. After connecting the sensor to the PC via powered USB, and supplying 20-24 V DC power to the unit, connect to the device by typing in the correct serial address (COM4 in Fig. 8) and click "Connect". The blank/background sample should then be loaded into the sample holder, and the desired number of 0.1-s readings to average over should be specified. To 
acquire a background measurement, click "Background". The value of the background measurement and the standard deviation are displayed in the software's graphical user interface (GUI). Load a test sample and click "Measure once" to acquire a single measurement; a checkbox determines whether or not background subtraction is carried out. Clicking "Start Continuous" causes the data stream to be continuously plotted within the GUI over a user-defined time range. Checking the "Record" checkbox causes the data stream to be saved to a comma separated variable (CSV) file; data is recorded both with and without a background subtraction. The continuous measurement can be interrupted at any time for a single measurement or a new background acquisition.

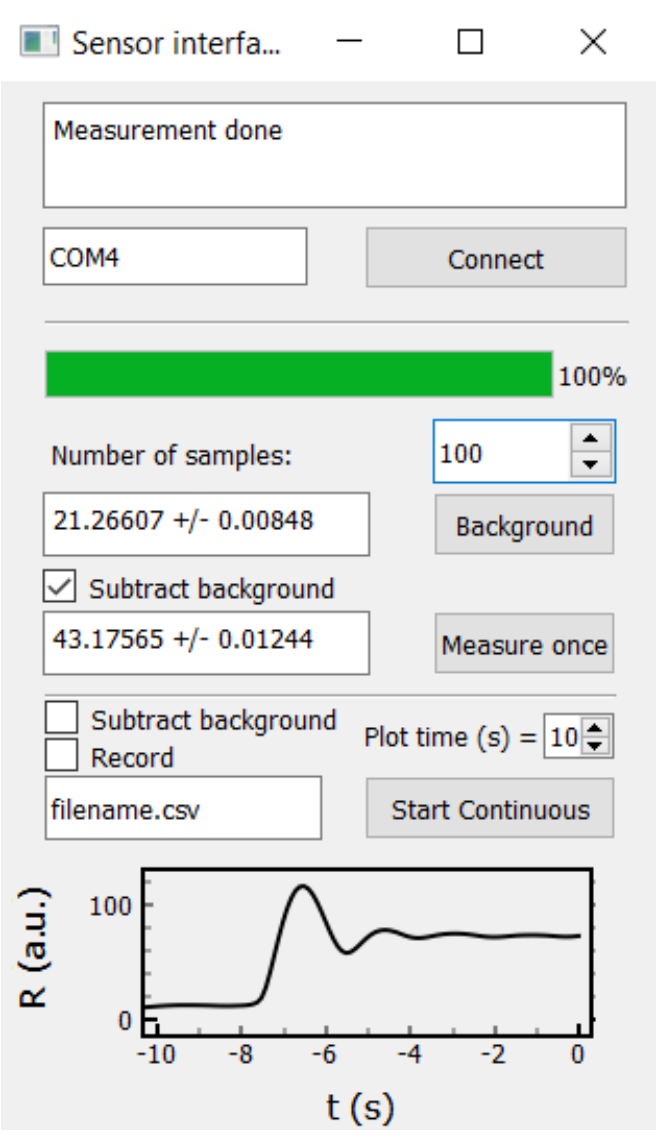

Fig. 8. Screenshot of GUI software used for displaying measurements.

\section{Validation and Characterisation}

Fig. 9 shows a "blank" signal $R_{2}^{\mathrm{b}}$ versus $t$ for a measurement obtained using pure water in the flow-cell, together with an equivalent "dark" signal $R_{2}^{\mathrm{d}}$ obtained under nominally identical conditions but with the LED disconnected. The two measurements were made under ambient conditions in a normally lit laboratory with no effort being made to shield the device from ambient light. The sample-rate and squarewave modulation frequency were set to the default values of $200 \mathrm{kHz}$ and $\sim 410 \mathrm{~Hz}$, respectively. $R_{2}^{\mathrm{d}}$ and $R_{2}^{\mathrm{b}}$ wandered around average values of -0.0009 and 22.6438 arbitrary units [a.u.] (see Fig. 9), respectively, with standard deviations $\sigma_{2}^{\mathrm{d}}$ and $\sigma_{2}^{\mathrm{b}}$ of 0.0014 a.u. and 0.0021 a.u. Neither signal showed signs of 
systematic drift. The higher value of the blank signal is attributable to a combination of leakage of excitation light through the long-pass filter and filter auto-fluorescence, and could be substantially reduced by switching to an orthogonal detection geometry and/or using a better optimised combination of short- and long-pass filters to further attenuate the unwanted light, see Fig. S1b. $R_{2}^{\mathrm{b}}$ represents a background signal that should be subtracted from any fluorescence measurement.
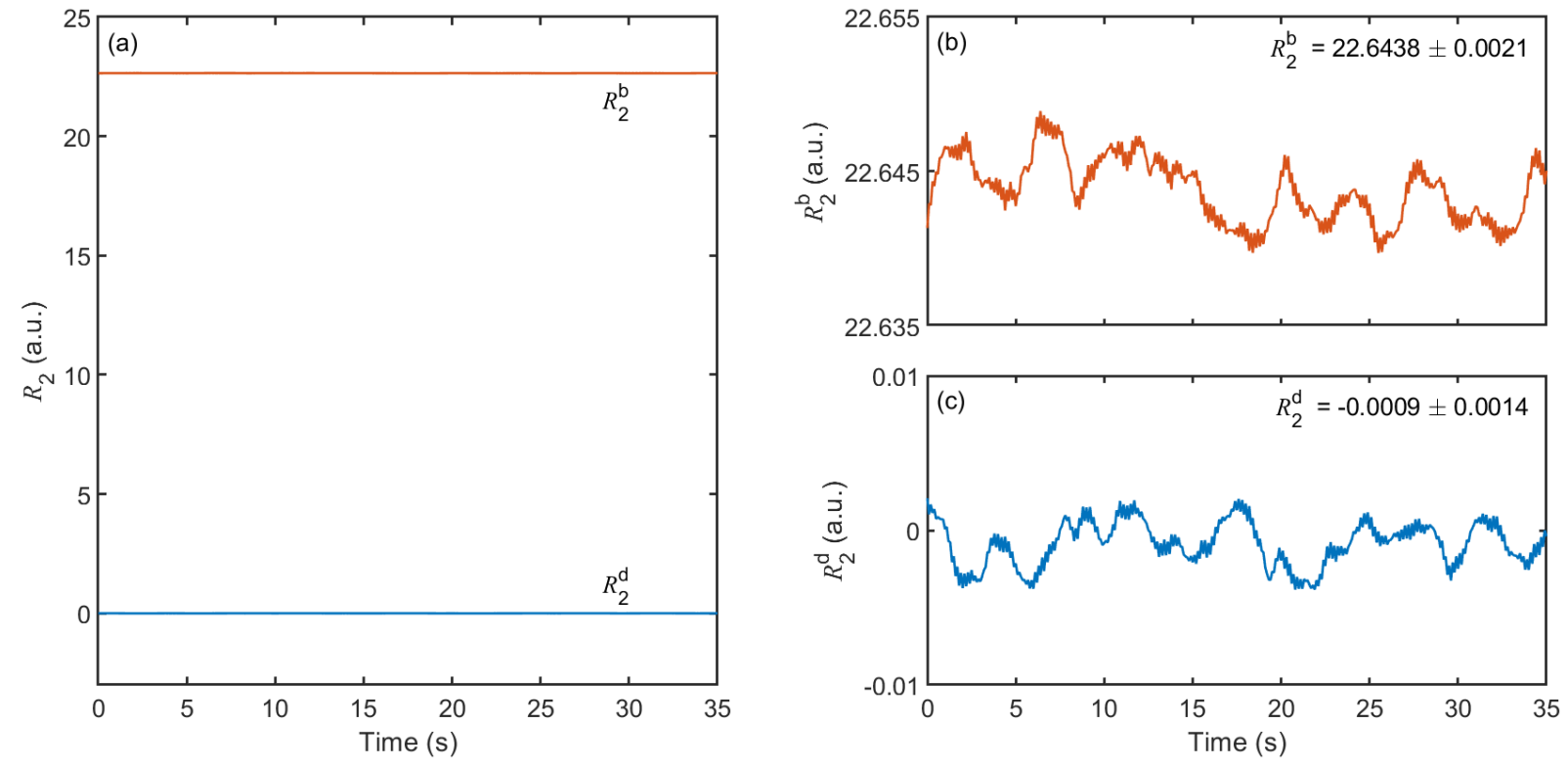

Fig. 9. (a) Blank $\left(R_{2}^{\mathrm{b}}\right)$ and dark $\left(R_{2}^{\mathrm{d}}\right)$ signals recorded using the fluorescence detector with pure water in the flow-cell. (b), (c) zoomed-in traces of $R_{2}^{\mathrm{b}}$ and $R_{2}^{\mathrm{d}}$; inset numbers denote mean plus or minus one standard deviation.

Fig. 10 shows the baseline-corrected signal $R_{2}^{\text {cor }}=R_{2}-R_{2}^{\mathrm{b}}$ versus concentration for a serial dilution of the fluorescent molecule resorufin from $0.35 \mathrm{mM}$ down to $54 \mathrm{nM}$. For each measurement, a solution of the dye molecule in $\mathrm{pH}-7.2$ phosphate-buffered saline was injected into the flow-cell using a syringe, and the $R_{2}$-value was recorded for ten seconds (after stabilisation) to obtain the mean and standard deviation $\sigma_{2} . R_{2}^{\text {cor }}$ varied linearly with the concentration $c$ of resorufin up to $c=39 \mu \mathrm{M}$, above which selfquenching led to a sub-linear response. $R_{2}$ could not be distinguished from the blank signal $R_{2}^{\mathrm{b}}$ at the lowest tested resorufin concentration of $54 \mathrm{nM}$. Least-squares fitting of the data to a line of the form $R_{2}^{\text {cor }}=m c$ indicated a sensitivity $m$ of $3.50 \pm 0.05 \times 10^{5}$ a.u./M, while extrapolation to $R_{2}^{\text {cor }}=2 \sigma_{2}$ implied a $2 \sigma$ detection limit $R_{2}^{\text {cor* }}$ of $65 \mathrm{nM}$. 


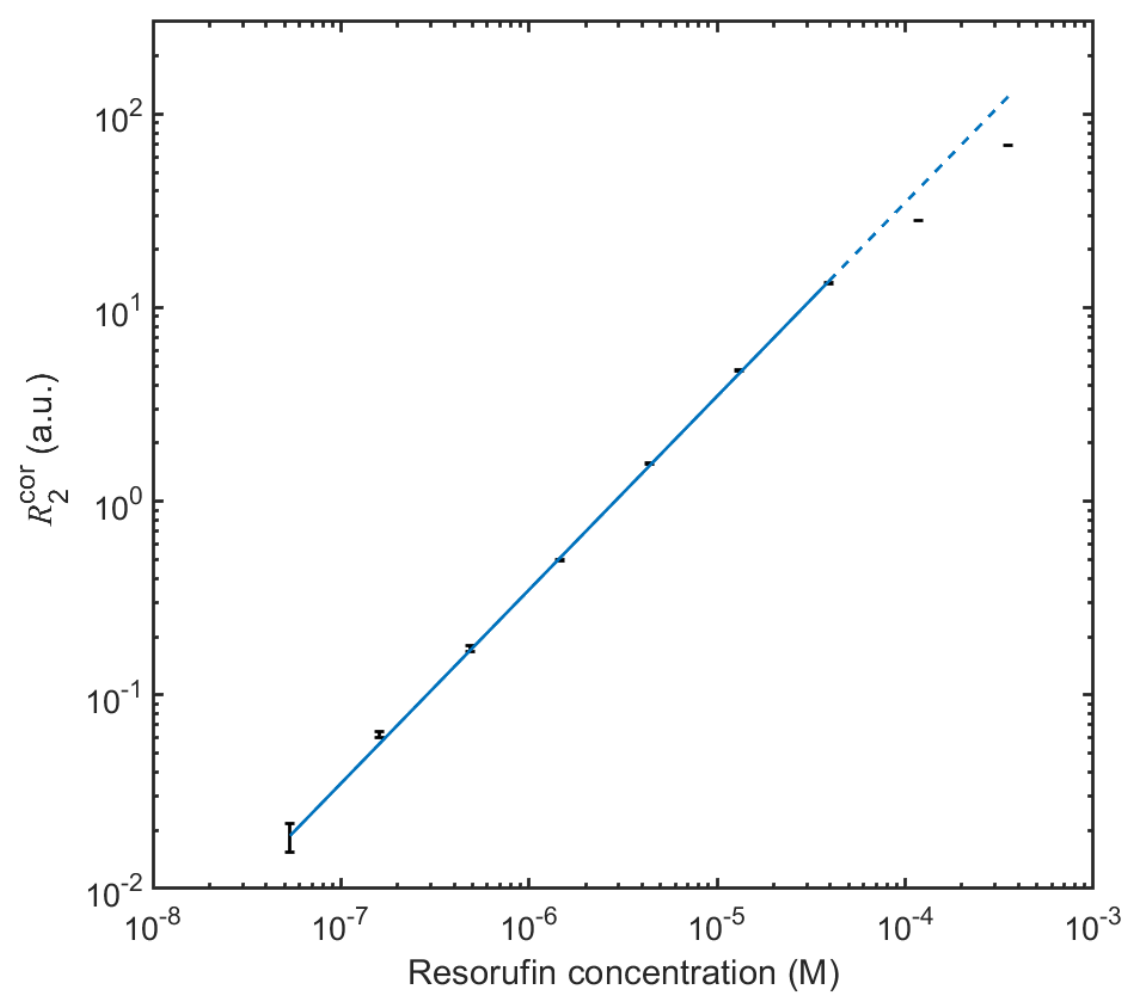

Fig. 10. Background-corrected signal $R_{2}^{\text {cor }}=R_{2}-R_{2}^{\mathrm{b}}$ versus resorufin concentration $c$ over the range $54 \mathrm{nM}$ to $0.35 \mathrm{mM}$, obtained using the flow-cell. The straight line represents a linear least-squares fit over the range $54 \mathrm{nM}$ to $39 \mu \mathrm{M}$ with an $\mathrm{R}^{2}$ value of 0.9990 . Above $39 \mu \mathrm{M}$ the relationship is sublinear due to the onset of dimerisation-induced self-quenching. The $2 \sigma$ detection limit $R_{2}^{\text {cor* }}$ is $81 \mathrm{nM}$. Error bars span mean corrected signal plus or minus one standard deviation.

Fig. 11 shows equivalent data to Fig. 10, obtained using a 10-mm pathlength glass cuvette in place of the flow-cell. The extended pathlength provided by the cuvette resulted in a 2.7-fold increase in sensitivity to $9.47 \pm 0.01 \times 10^{5}$ a.u./M, with successful detection down to $18 \mathrm{nM}$ and an estimated $2 \sigma$ detection limit $R_{2}^{\text {cor* }}$ of $18 \mathrm{nM}$. Dividing the largest concentration in the linear range $(0.35 \mathrm{mM})$ by the smallest detectable concentration (18 $\mathrm{nM}$ ) indicated a dynamic range of approximately $2 \times 10^{4}$, sufficient for most diagnostic applications.[10-12] 


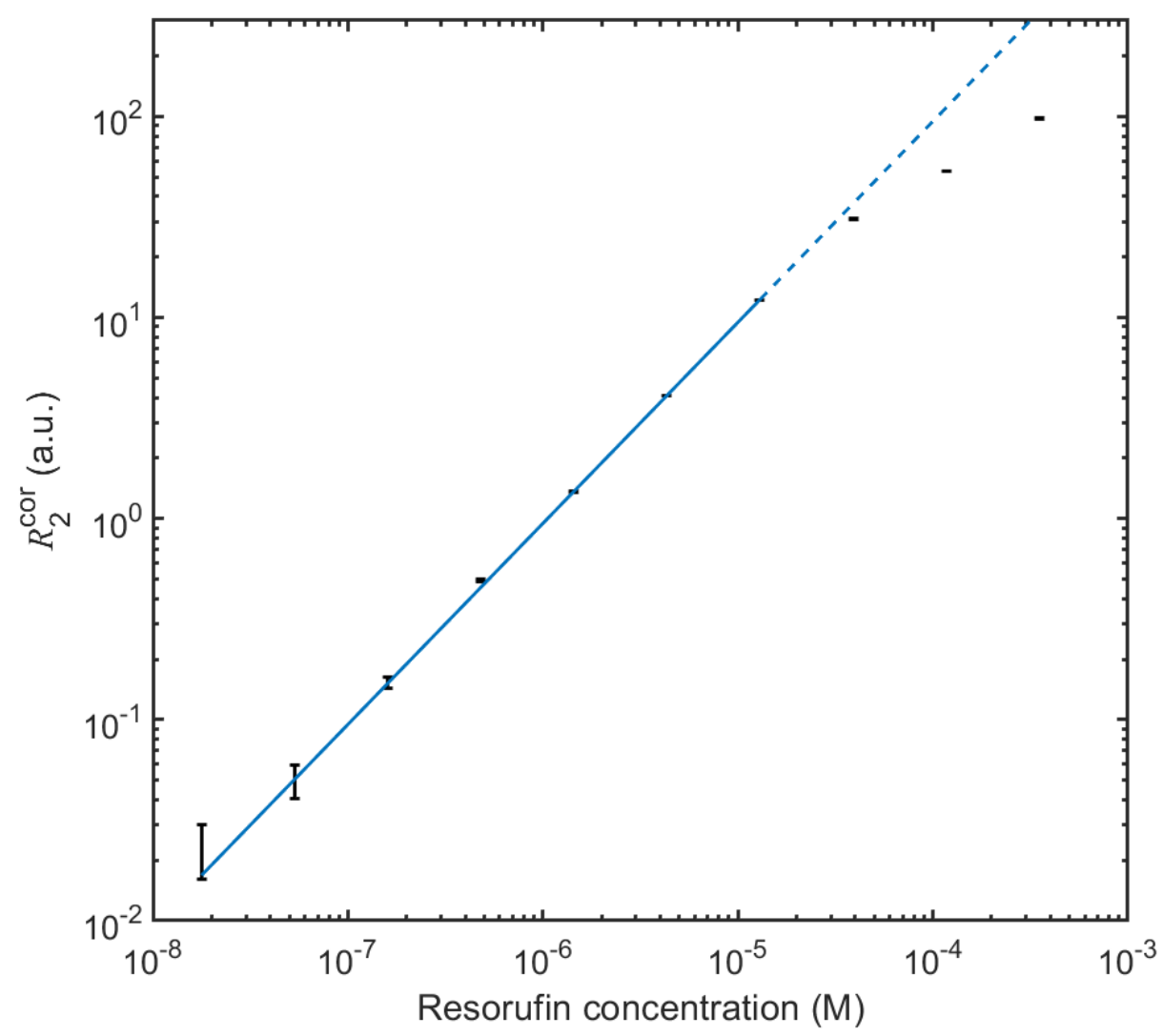

Fig. 11. Background-corrected signal $R_{2}^{\text {cor }}=R_{2}-R_{2}^{b}$ versus resorufin concentration $c$ over the range $18 \mathrm{nM}$ to $0.35 \mathrm{mM}$, obtained using a 10-mm pathlength cuvette in the optical stack. The straight line represents a least-squares linear fit over the range $18 \mathrm{nM}$ to $13 \mu \mathrm{M}$ with an $\mathrm{R}^{2}$ value of 1.0000 . Above $13 \mu \mathrm{M}$, a sublinear relationship is observed due to selfquenching and internal filtering of resorufin in the solution. The $2 \sigma$ detection limit is $18 \mathrm{nM}$. Error bars span mean corrected signal plus or minus one standard deviation.

The detector was further applied to the detection of cholesterol, using a fluorimetric assay based on the widely used probe Amplex Red (see Fig. 12a). The enzyme cholesterol oxidase catalyses the oxidation of cholesterol to cholestenone, with the concurrent reduction of oxygen to hydrogen peroxide $\left(\mathrm{H}_{2} \mathrm{O}_{2}\right)$. In the presence of horseradish peroxidase, the generated $\mathrm{H}_{2} \mathrm{O}_{2}$ converts Amplex Red to the highly fluorescent dye molecule resorufin, with the measured fluorescence intensity being directly proportional to the initial cholesterol concentration. A stock solution of $4 \mathrm{U} / \mathrm{ml}$ cholesterol oxidase, $4 \mathrm{U} / \mathrm{ml}$ horseradish peroxidase (HRP), and $300 \mu \mathrm{M}$ Amplex red was made up in PBS with 1\% (v/v) TritonX-100. Six 1-ml aliquots were taken from the stock solution and combined with 1-ml cholesterol solutions with concentrations ranging from $780 \mathrm{nM}$ to $25 \mu \mathrm{M}$. A negative control sample of $1 \%$ TritonX-100 in PBS (without cholesterol) was also prepared, and was used as the "blank" sample. The cholesterol solutions were prepared in PBS and

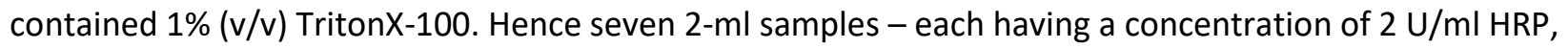
$2 \mathrm{U} / \mathrm{ml}$ cholesterol oxidase and $150 \mu \mathrm{M}$ Amplex red - were obtained with cholesterol concentrations ranging from 0 to $12.5 \mu \mathrm{M}$ cholesterol. The samples were left for 30 minutes at room temperature for the 
reaction to complete, and were then loaded sequentially into the flow-cell to determine the fluorescence intensity. For each sample the lock-in amplitude $R_{2}$ was recorded for 10 seconds after stabilisation.

Fig. $12 \mathrm{~b}$ shows the baseline-corrected signal $R_{2}^{\text {cor }}=R_{2}-R_{2}^{\mathrm{b}}$ versus cholesterol concentration $c$ where $R_{2}^{\mathrm{b}}$ is the background signal obtained with the negative control sample. Least-squares fitting of the data to a line of the form $R_{2}^{\text {cor }}=m c$ indicated a sensitivity $m$ of $9.2 \times 10^{4}$ a.u./M with an $R^{2}$ linear correlation coefficient of 0.9984 , while extrapolation to $R_{2}^{\text {cor }}=2 \sigma_{2}^{\mathrm{b}}$ implied a detection limit $R_{2}^{\text {cor* }}$ of $200 \mathrm{nM}$-some 25000 times lower than the typical 5-mM concentration of cholesterol in human serum (and hence sufficient for diagnostic applications). [10,11]

(a)

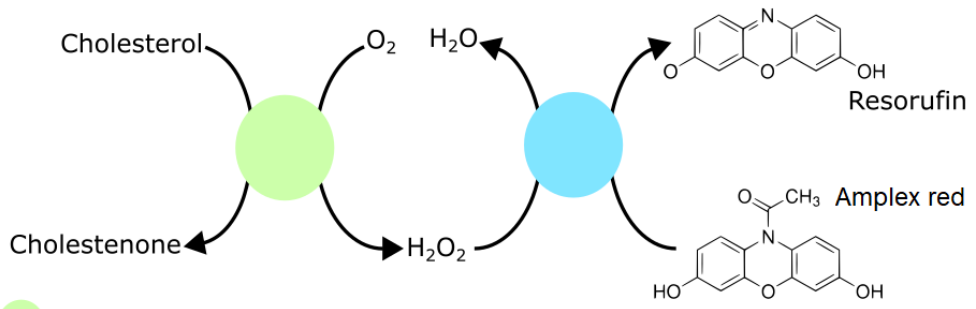

- Cholesterol oxidase

- Horseradish peroxidase

(b)

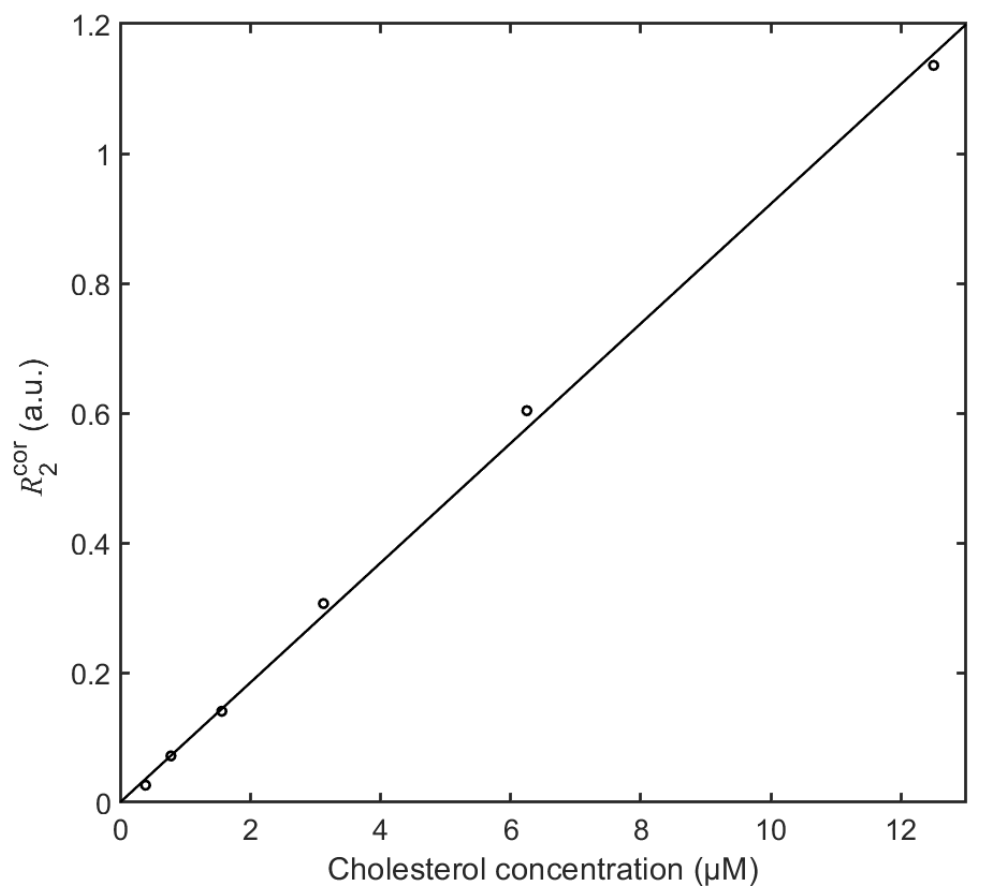

Fig. 12. (a) Scheme showing chemical procedure used for cholesterol detection: cholesterol is oxidised by cholesterol oxidase to produce $\mathrm{H}_{2} \mathrm{O}_{2}$, which in turn is used by horseradish peroxidase (HRP) to convert Amplex Red into the fluorescent dye resorufin. (b) Corrected signal $R_{2}^{\text {cor }}=R_{2}-R_{2}^{b}$ vs cholesterol concentration $c$ for the HRP assay. The straight line represents a least-squares optimised linear fit with $\mathrm{R}^{2}=0.9984$ and a $2 \sigma$ detection limit of $200 \mathrm{nM}$. 


\section{Declaration of competing interests}

There are no conflicts of interest to declare.

\section{References}

[1] D.M. Amundson, M. Zhou, Fluorometric method for the enzymatic determination of cholesterol, J. Biochem. Biophys. Methods. 38 (1999) 43-52. https://doi.org/10.1016/S0165-022X(98)000360.

[2] L.-M. Bai, H. Zhou, W.-E. Liu, H. Chai, L.-P. Yang, W. Yan, W. Zhang, H.-H. Yang, W. Jiang, Fluorescent monitoring of the reaction kinetics of nonfluorescent molecules enabled by a fluorescent receptor, Chem. Commun. 55 (2019) 3128-3131. https://doi.org/10.1039/C9CC00762H.

[3] L. Tang, L. Zhou, A. Liu, X. Yan, K. Zhong, X. Liu, X. Gao, J. Li, A new cascade reaction-based colorimetric and fluorescence "turn on" dual-function probe for cyanide and hydrazine detection, Dye. Pigment. 186 (2021) 109034. https://doi.org/10.1016/j.dyepig.2020.109034.

[4] A.M.A. Adam, T.A. Altalhi, S.M. El-Megharbel, H.A. Saad, M.S. Refat, I. Grabchev, R.A. Althobaiti, Detection of environmental pollutants heavy metal ions based on the complexation with fluorescent dyes: Reaction of 2-(2'-hydroxyphenyl)-5-amino-benzotriazole with the $\mathrm{Sn2}+, \mathrm{Hg} 2+$, and $\mathrm{Pb} 2+$ ions, Inorg. Chem. Commun. 124 (2021) 108408.

https://doi.org/10.1016/j.inoche.2020.108408.

[5] Q. Han, X. Liu, X. Wang, Y. Song, L. Yang, J. Li, T. Qiang, Photoactivated fluorescence-based analysis for the facilitative and selective detection of silver(I) in aqueous solutions, Dye. Pigment. 184 (2021) 108793. https://doi.org/10.1016/j.dyepig.2020.108793.

[6] K. Kishore, S.A. Akbar, Evolution of Lock-In Amplifier as Portable Sensor Interface Platform: A Review, IEEE Sens. J. 20 (2020) 10345-10354. https://doi.org/10.1109/JSEN.2020.2993309.

[7] R.G. Lyons, Understanding digital signal processing, 3rd ed., Prentice Hall Publishing, 2011.

[8] M. Yamazaki, S. Krishnadasan, A.J. DeMello, J.C. DeMello, Non-emissive plastic colour filters for fluorescence detection, Lab Chip. 12 (2012) 4313. https://doi.org/10.1039/c2lc40718c.

[9] M. Yamazaki, O. Hofmann, G. Ryu, L. Xiaoe, T.K. Lee, A.J. DeMello, J.C. DeMello, Non-emissive colour filters for fluorescence detection, Lab Chip. 11 (2011) 1228. https://doi.org/10.1039/c0lc00642d.

[10] M.A. Al-Rawhani, B.C. Cheah, A.I. Macdonald, C. Martin, C. Hu, J. Beeley, L.C. Gouveia, J.P. Grant, G. Campbell, M.P. Barrett, D.R.S. Cumming, A Colorimetric CMOS-Based Platform for Rapid Total Serum Cholesterol Quantification, IEEE Sens. J. 17 (2017) 240-247. https://doi.org/10.1109/JSEN.2016.2629018.

[11] V. Narwal, R. Deswal, B. Batra, V. Kalra, R. Hooda, M. Sharma, J.S. Rana, Cholesterol biosensors: A review, Steroids. 143 (2019) 6-17. https://doi.org/10.1016/j.steroids.2018.12.003.

[12] A. Gahlaut, V. Hooda, V. Dhull, V. Hooda, Recent approaches to ameliorate selectivity and sensitivity of enzyme based cholesterol biosensors: a review, Artif. Cells, Nanomedicine Biotechnol. 46 (2018) 472-481. https://doi.org/10.1080/21691401.2017.1337028. 